\title{
Transcervical approach for left ventricular assist device outflow graft: A proof-of-concept implantation study
}

\author{
Fraser William Havern Sutherland, MA, MB, BChir, MD(Cantab), FRCS, George Gradinariu, MD, MRCS, \\ and Philip Curry, MB, BS, FRCS, Glasgow, United Kingdom
}

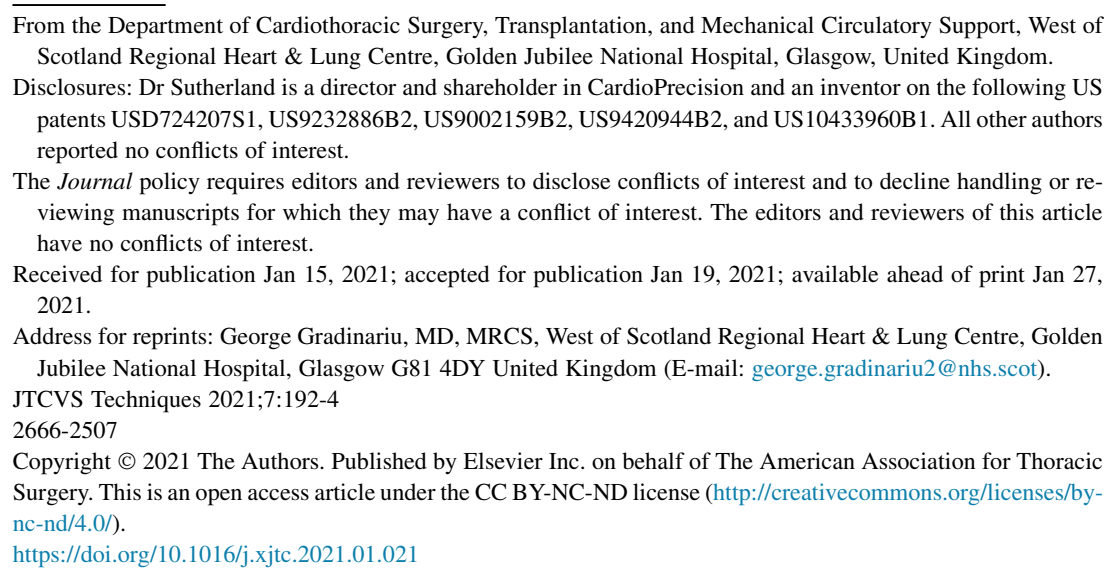

$\checkmark$ Video clip is available online.

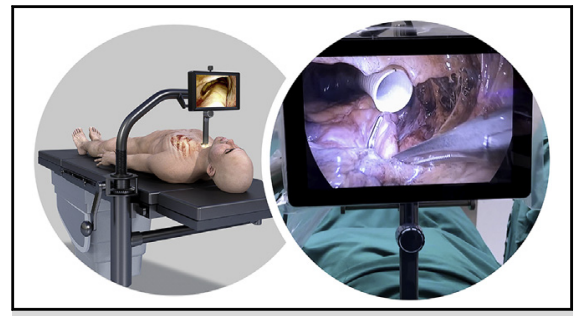

Transcervical approach to outflow graft anastomosis using the CoreVista Retractor.

CENTRAL MESSAGE

Our technique demonstrates the proof-of-concept for a novel

minimalist transcervical

approach for ascending aortic anastomosis of a left ventricular assist device outflow graft.

See Commentaries on pages 195 and 197.
Minimally invasive techniques to implant centrifugal left ventricular assist devices (LVADs) have been described as alternatives to the classic full sternotomy approach. Implantation of the pump is done through a left anterior thoracotomy. The outflow graft is anastomosed to the ascending aorta via upper hemisternotomy or right anterior thoracotomy. 1,2

The goal is to decrease periprocedural bleeding, achieve early mobilization, reduce in-hospital stay, and facilitate future sternotomies in patients where assist devices are used as a bridge to transplantation.

We hypothesized that the outflow graft anastomosis could be achieved through a minimalist transcervical incision avoiding a sternotomy/right thoracotomy. This report shows evidence to support this hypothesis.

\section{SURGICAL TECHNIQUE}

This was a proof-of-concept implantation performed on a cadaveric model embalmed via the Thiel technique, which optimally preserves the consistency, color, transparency, and flexibility of tissues making it a practical model for assessing vascular surgical techniques. ${ }^{3}$
Graft anastomosis is facilitated by a novel access system (CoreVista, CardioPrecision Ltd, Glasgow, United Kingdom) specifically designed for cardiothoracic surgery via the minimalist transcervical approach. This device comprises a frame fixed to the operating table to which is attached a single-use retractor with in-built illumination used for the different steps of the procedure. A highdefinition surgical monitor is attached to the lifting arm of the frame and its position adjusted for optimal viewing by the surgeon. The monitor is encased in a sterile, disposable drape with optically clear central window to allow the graft anastomosis to be performed onscreen using long-shafted minimally invasive cardiac surgery instruments (Figure 1).

The insertion of the centrifugal pump and tunneling of the outflow graft are well established and were not replicated in the cadaver. The technique for outflow graft anastomosis can be broken down in a number of reproducible and easy-to-perform steps. Firstly, a simple transverse skin crease incision is done at the base of the neck and soft tissues immediately behind the sternum and under each sternoclavicular joint are mobilized with electrocautery. The sternohyoid muscles are detached from the 


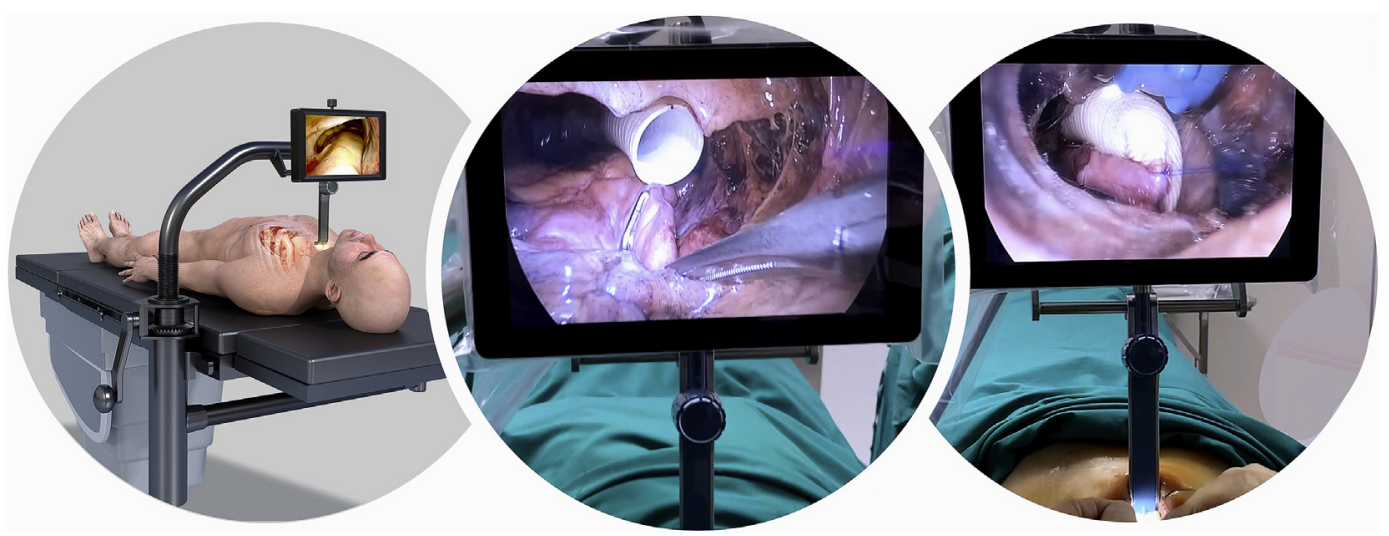

FIGURE 1. Minimalist transcervical approach for left ventricular assist-device outflow graft anastomosis. CoreVista Retractor (CardioPrecision Ltd, Glasgow, United Kingdom) and accessories (left panel), on-screen view of graft ready for anastomosis (middle panel), and on-screen view of completed anastomosis (right panel).

posterior surface of the sternum. Following introduction of the retractor and elevation of the sternum, the brachiocephalic artery is identified and followed into the chest. The innominate vein is identified and the pericardium incised just caudal to this level. The ascending aorta is then exposed and the outflow graft pulled upward. After measuring, trimming, and orienting the graft, a sideclamp is used to isolate the best anastomotic site on the ascending aorta. The aorta is incised and the outflow graft sutured in a continuous fashion using 4-0 Prolene sutures starting at the heel and working toward the surgeon on each side.

The anastomosis is optimally performed onscreen using minimally invasive cardiac surgery instruments and a Derra-Cooley clamp. The specially designed illumination provided by the CoreVista Retractor as well as the in-built high-definition monitor in the line of the incision greatly facilitates surgery (Video 1). Once the anastomosis is com-

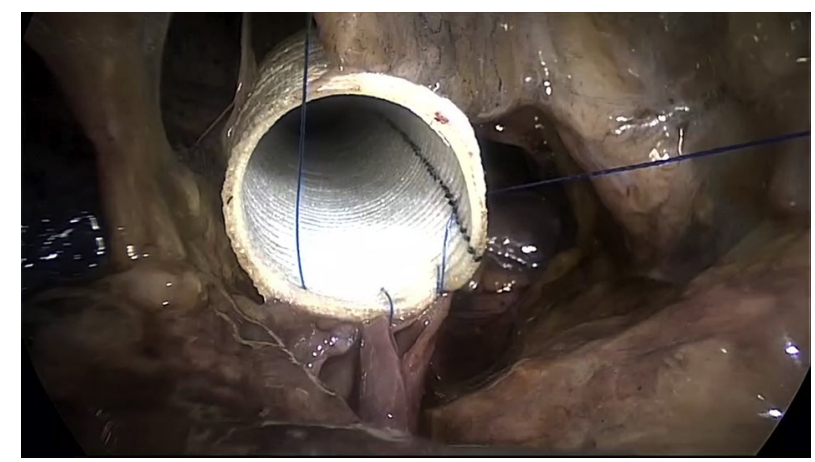

VIDEO 1. Setup and technical steps for our minimalist transcervical approach for a left ventricular assist-device outflow graft anastomosis performed on a Thiel-preserved cadaver using the CoreVista Retractor (CardioPrecision Ltd, Glasgow, United Kingdom) and its accessories. Video available at: https://www.jtcvs.org/article/S2666-2507(21)00089-4/ fulltext. plete, de-airing is performed either through the toe of the graft or via a root vent placed into the ascending aorta beyond the graft. Sutures are hand tied and the clamp released. The orientation of the graft is inspected with the aid of the thoracoscopic camera. Hemostasis is readily achievable through this approach, as demonstrated previously in transcervical surgical aortic valve replacement (SAVR). ${ }^{4}$

\section{DISCUSSION}

A trial investigating the lateral thoracotomy implant approach for a centrifugal-flow LVADs could decrease bleeding complications and length of stay. ${ }^{5}$ The main advantage of our new transcervical approach, performed on- or off-pump, is that it completely avoids the hemisternotomy or right-sided thoracotomy. ${ }^{1,2}$ This not only avoids the pain and bleeding complications of full or partial sternotomy but also eliminates the impaired lung function and potentially difficult access associated with right-sided thoracotomy. The aortic arch is also accessible for cannulation with this approach. This technique should also minimize the risk for the well-described right ventricle decompensation that is precipitated by full opening of the pericardium.

This minimalist transcervical approach has already proved feasible for transcervical transcatheter aortic valve replacement and SAVR in live patients, the latter with endotracheal tube, transesophageal echocardiography probe, and central lines in situ. ${ }^{4}$ Given the much higher complexity of SAVR, we believe that, after an initial learning curve, using this approach should be easy and reproducible.

\section{Study Limitations}

The main limitation is the use of the cadaveric model. Although this aims to replicate the anatomical conditions 
encountered in a live patient, some differences remain in patients undergoing surgery.

\section{CONCLUSIONS}

Our proof-of-concept implantation study illustrates a novel approach to LVAD outflow graft anastomosis that completely avoids sternotomy or thoracotomy.

\section{References}

1. Schmitto JD, Krabatsch T, Damme L, Netuka I. Less-invasive HeartMate 3 left ventricular assist device implantation. J Thorac Dis. 2018;10:S1692-5.
2. Ayers B, Sagebin F, Wood K, Barrus B, Thomas S, Storozynsky E, et al. Complete sternal-sparing approach improves outcomes for left ventricular assist device implantation in patients with history of prior sternotomy. Innov Technol Tech Cardiothorac Vasc Surg. 2020;15:51-6.

3. Healy SE, Rai BP, Biyani CS, Eisma R, Soames RW, Nabi G. Thiel embalming method for cadaver preservation: a review of new training model for urologic skills training. Urology. 2015;85:499-504.

4. Dapunt OE, Luha O, Ebner A, Sonecki P, Spadaccio C, Sutherland FWH. First-inman transcervical surgical aortic valve replacement using the corevista system. Innov Technol Tech Cardiothorac Vasc Surg. 2016;11:84-93.

5. McGee E, Danter M, Strueber M, Mahr C, Mokadam NA, Wieselthaler G, et al. Evaluation of a lateral thoracotomy implant approach for a centrifugal-flow left ventricular assist device: the LATERAL clinical trial. J Heart Lung Transplant. 2019;38:344-51. 